\title{
Image Guided Radiotherapy for Cervical Cancer: A Review
}

\author{
Rob A. Helms \\ University of Missouri-Kansas City, USA.
}

\begin{abstract}
How to cite this paper: Rob A. Helms. (2020) Image Guided Radiotherapy for Cervical Cancer: A Review. International Journal of Clinical and Experimental Medicine Research, 4(3), 53-57.

DOI: $10.26855 /$ ijcemr.2020.07.004
\end{abstract}

Received: April 27, 2020

Accepted: May 31, 2020

Published: June 24, 2020

\begin{abstract}
Cervical cancer (CC) is one of the most common cancers in female worldwide. Radiotherapy plays an important role in the treatment of cervical cancer, and about $80 \%$ of CC patients need radiotherapy. However, many factors, including body position, body mass index and bladder filling state and so on, can affect the set-up errors during the treatment of radiotherapy. Recently, image guided radiotherapy has been widely used in clinic to reduce set-up errors and improve the accuracy of radiotherapy. Besides, image guided radiotherapy is applied for the analysis of cervical cancer target expansion. There is no doubt that radiotherapy could improve the therapeutic effect of cervical cancer, but the complications caused by radiotherapy should also draw our attentions.
\end{abstract}

\section{Keywords}

Cervical cancer, set-up errors, BMI, bladder filling state, image guided radiotherapy

\section{Introduction}

Tumor is a common disease, which is caused by many factors. Recently, operation, radiotherapy and chemotherapy are the convention treatment methods of tumor in clinic.

Radiotherapy is very common among all the treatments, which can be used for the treatment of local tumor [1-3].

Ionizing radiation was discovered to be used to treat pituitary tumors as an adjunctive therapy [4]. Cell death is induced by radiotherapy through cellular level. Since the process takes a long time to normalize hormone levels, adjunctive medical therapies are required at the same time [5]. External beam radiation is most commonly applied for the treatments of pituitary adenomas with conventional radiotherapy (CRT) [6]. CT/MRI is involved in targeted treatment planning, besides, the customized mask is also used for stabilization of head and target of precise tumor. Stereotactic radiosurgery (SRS), including proton beam therapy and Gamma Knife, require neuroimaging techniques, for instance, CT/MRI or PET scan mapping [7].

The basic principle of precise radiotherapy for tumor is to ensure that the radiation can concentrate on the tumor area to the maximum extent, and the surrounding normal tissue can be well protected [8]. This requires accurate diagnosis, precise location, precise radiotherapy plan and precise treatment before radiotherapy treatment [9]. It is very important to improve the fitness of target area as well as the dose of target area. There are many uncertain factors that affect the accuracy of radiotherapy in the process of tumor radiotherapy, including the displacement of tumor and normal tissue, the positioning error in treatment, the reliability and stability of treatment equipment and other factors [10]. How to solve these problems and improve the accuracy of target irradiation is very essential in the field of radiotherapy [11].

Cervical cancer (CC) is one of the most common cancers in female worldwide. It's reported that there are 
98,900 new cases and 30,500 thousand deaths every year in China [12]. For postoperative patients with cervical cancer, IMRT is associated with comparable survival and decreased toxicity. Radiotherapy plays an important role in the treatment of cervical cancer, and about $80 \%$ of CC patients need radiotherapy [13]. Radiotherapy planning refers to the calculation of dose deposition throughout the patient, based upon quantitative electron density images from computed tomography (CT) scans taken before treatment. Cone beam CT (CBCT), consisting of a point source and flat panel detector, is often built onto radiotherapy delivery machines and used during a treatment session to ensure alignment of the patient to the plan [14]. There are many factors that can affect the accuracy of radiotherapy target area in the process of CC radiotherapy. In addition to the inherent error of the system, the positioning error, the involuntary movement of human organs and the displacement caused by different filling states, and the change of tumor volume in the treatment are the common causes of radiotherapy error [15].

\section{Application of image guided radiotherapy in the analysis of cervical cancer set-up error}

The set-up error can be divided into system error and random error. The precision of machine is the mainly cause of system error, while random error is resulted from the movement of tissues and organs, positioning technology as well as the performance of radiotherapy doctors [16]. System error refers to the difference between the position of patients in the treatment of different times and the position in the simulation positioning. It's caused by the inconsistency of the machine system itself, such as the inconsistency between the accelerator and the simulator, the error between the accelerator and the simulation positioning, etc. It's possible to reduce the system error by regular quality assurance and quality control of the machine [17]. However, random error refers to the difference of position repeatability of patients during each treatment, which occurs during the actual treatments. Random error is related to the operation of technicians, the position maintenance of patients and the movement of organs [18]. Therefore, we pay attentions to random error in clinic because it significantly affects the treatment effect.

\section{Set-up error in image guided radiotherapy of cervical cancer}

Two kinds of set-up errors occur in treatment: random error and systematic error. Random error may lead to the change of dose distribution, which will cause the decrease of local control rate of tumor or the increase of complications of normal tissue. Therefore, we should take random error into consideration and try to minimise error and improve the accuracy during the treatment [19].

Image guided radiation therapy (IGRT) is a new radiotherapy technology following three-dimensional conformal radiotherapy and intensity-modulated radiotherapy. According to classification of image dimension, image guided radiotherapy technology can be divided into a number of categories, including two-dimensional KV image, MV image and three-dimensional KV-CBCT image or MV-CBCT image. Image guided radiotherapy can be used to analyze the location of tumor, the size of target area, the changes of organs at risk and the changes of bladder. Therefore, image guided radiotherapy has been widely used in the radiotherapy of cervical cancer [20-21].

\subsection{Body position and Body mass index affect the set-up error}

Because of less rigid structure, more subcutaneous fat, soft and deformable abdominal wall, the effect of body position fixation is often inferior to that of head and neck tumor. As stated in Jian Xu et al.'s study, the volume of dangerous organs around cervical cancer, such as bladder and small intestine, changes with the filling degree and body position. Thus, how to improve the positioning accuracy through reasonable body position and fixation is particularly important [22]. According to Zhang et al.'s research, vacuum bag immobilization caused the least set-up errors compared with thermoplastic body model immobilization, while body model immobilization could reduce the position error compared with no immobilization $(\mathrm{P}<0.01)$ [23]. In Ping Li et al.'s study, pre-and post-fraction CBCT scans were used for 18 cervical cancer patients. The moving distance of center point in tumor was (8. 890 \pm 7.222$) \mathrm{mm}$ before CBCT, and after correction the distance was $(1.021 \pm 1$. 075) $\mathrm{mm}$, the result indicated that CBCT scans could significantly reduce setup errors after correction [24]. As stated in Liuetal's study, no significant difference was found between supine position and prone position if the body mass index (BMI) was not been taken into consideration. For the patients with $\mathrm{BMI}<24 \mathrm{~kg} / \mathrm{m}^{2}$, the setup errors of supine position were significantly more than those of prone position in $\mathrm{Z}$ direction, while for the patients with BMI $>24$ $24 \mathrm{~kg} / \mathrm{m}^{2}$, the setup errors of supine position were significantly less than those of prone position in $\mathrm{Z}$ direction. Besides, no significant difference was observed between the two groups in X and Y direction [25]. Yi Liu et al. 
also pointed out that the set-up errors of $\mathrm{X}$-axis, $\mathrm{Y}$-axis and Z-axis in the normal group was significant less than that in the obese group before correction. The result also indicated that CBCT can effectively corrected the positioning error and was not affected by the patient's BMI [26]. Cai et al. pointed out that the setup error of obese patients is larger. The application of CBCT image guided technology in setup error correction could reduce the impact of patient's body shape on MPTV value [27].

Obese patients lie down with skin traction on the surface. The thicker the subcutaneous fat layer, the more severe the skin pull, especially in patients with thick abdominal fat and loose skin. Therefore, the patients must avoid the left and right movement as much as possible to avoid local skin traction. The set-up errors might be related to the patients breathing, especially for the abdominal breathing. Since obese patients have a relatively thick body, and the amplitude of respiratory movement on the body surface will be larger than that of normal patients. It will be better for the patients to breathe steadily and adopt shallow breathing and chest breathing as much as possible.

\subsection{Bladder filling state affects set-up error}

Cervical cancer is a common malignant tumor of female reproductive system. With the progress of medicine and the prolongation of patients' survival time, the quality of life evaluation after treatment has become an important standard for quality methods [28]. IMRT (intensity modulated radiation therapy) can improve the accuracy of radiotherapy target area of cervical cancer, reduce radiation exposure to normal tissues and organs, reduce the incidence of complications, and improve the quality of life of patients, however, in the course of radiotherapy, with the tumor shrinking, the tumor volume and location may change dramatically. When the bladder is involved in the radiotherapy, the incidence of acute and chronic complications of the digestive system may occur [29].

The bladder is located in the center of the pelvic cavity. It is a kind of variable cystic organ. The volume, size and shape of bladder are determined by the filling degree. In general, the capacity of adult bladder is about $300-500 \mathrm{ml}$, and the maximum capacity is $800 \mathrm{ml}$. With the increase of bladder volume, the small intestine area can be pushed upward by the full bladder, and then the treatment irradiation area can be changed. When the bladder becomes smaller, the empty part is easily filled by the small intestine, and the treatment irradiation area will be changed as well. Reliability of bladder volume at the time of radiation treatment is low, regardless of bladder filling instructions, although an empty bladder reduces absolute variation in bladder volume. The filling degree of the bladder determines the change of the volume and position structure of the bladder, which may have a certain impact on the absorbed dose [30-32]. Besides, different doctors will also have different ways to sketch the bladder boundary, which may lead to the error of bladder volume [33]. Bladder filling can not only reduce the radiation dose of the bladder itself, but also the radiation dose of the small intestine [34]. Therefore, it's very important to ensure the fullness of bladder during the treatment.

\section{Application of image guided radiotherapy in the analysis of cervical cancer target expansion}

Before making the radiotherapy plan for cervical cancer, the tumor area and normal tissue should be sketched first. The tumor area (GTV) includes tumor focus and metastatic lymph nodes, while clinical target volume (CTV) includes tumor area, subclinical focus and possible invasion area. However, for the patients after the operation, CTV was needed to sketch [35, 36]. But at present, there is no uniform standard for CTV. Different units have different descriptions of target areas and organs.

The regression of CTV during radiotherapy will also affect the absorbed dose of bladder, while the larger CTV volume will obviously squeeze the bladder, resulting in the deformation of bladder volume and the displacement of geometric center, while the absorbed dose of large CTV target area to bladder will exceed 50\% of V45 [37]. Therefore, it is necessary to closely monitor the patients with large CTV during radiotherapy, and modify the patient's plan and recalculate the evaluation dose in time [38].

For the normal tissues outlined, including rectum, bladder, small intestine and femoral head, some hospitals and units include bone marrow to reduce blood toxicity. It may cause the movement of the target area due to the movement of the organs in the pelvic tissue, deformation, regression and reduction of mass. How to avoid the impact of these factors, planning target volume (PTV) is conducted in this process [39, 40]. Uterus movements are large and extremely variable among patients. They carry uncertainties for the CTV coverage and justify consequent margins for the PTV, thus limiting the benefit of IMRT. According to the previous studies, a minimum of $10 \mathrm{~mm}$ around the centro-pelvic CTV (with 10 to $15 \mathrm{~mm}$ in anterior-posterior direction) and 7 to 10 $\mathrm{mm}$ around the nodal CTV seem to be a reasonable compromise for nonadaptive intensity-modulated pelvic 
radiotherapy [41].

\section{Conclusion}

In summary, the focus of high-risk cervical cancer on early stage is occult and easy to recur and metastasize. The clinical effect of surgery is poor, while postoperative adjuvant radiotherapy can eliminate the hidden focus of pelvic cavity and reduce the recurrence rate of pelvic cavity. Radiotherapy has been widely used after radical operation of cervical cancer. Image guided radiotherapy of cervical cancer can improve the accuracy of radiotherapy for cervical cancer patients. The development of image registration and fusion technology plays an increasingly important role in image-guided radiotherapy of tumor, which is crucial for the accuracy of target area and critical organ delineation. As the main treatment method of cervical cancer, there is no doubt that radiotherapy could improve the therapeutic effect of cervical cancer, but the complications caused by radiotherapy are still a problem that cannot be ignored in clinical.

\section{Conflict}

The authors declare no conflict of interest.

\section{References}

[1] Miyahira AK, Den RB, Carlo MI, et al. (2019). Tumor cell heterogeneity and resistance; report from the 2018 Coffey-Holden Prostate Cancer Academy Meeting [J]. The Prostate, 79(3): 244.

[2] H. Shuang, J. Feng, C. Caineng. (2019). The value of radical radiotherapy in the primary tumor of newly diagnosed oligo-metastatic nasopharyngeal carcinoma patients [J]. Clinical \& Translational Oncology, 21(2): 213-219.

[3] Xinhe Liu, Ying Liu, Junning Wang. (2019). Mild Hyperthermia-Enhanced Enzyme-Mediated Tumor Cell Chemodynamic Therapy [J]. ACS Applied Materials \& Interfaces, 11(26).

[4] Mingyin Jiang, Linlin Wang, Jiaqi Gao. (2019). Risk Analysis of Radiotherapy Implementation Process Based on Failure Mode and Effect Analysis [J]. Chinese Journal of Medical Instrumentation, 43(3): 230-234.

[5] Guo, CL (Guo Chuanling), Wang, JF (Wang Jufang), Jin, XD (Jin Xiaodong), et al. Studies on advantages of heavy ions in radiotherapy compared with gamma-rays [J]. Nuclear Inst \& Methods in Physics Research B, 3923, 259(2): 997-1003.

[6] Al Sajwani A., Sherlock M. (2019). Radiotherapy. In: Llahana S., Follin C., Yedinak C., Grossman A. (eds) Advanced Practice in Endocrinology Nursing. Springer, Cham.

[7] Kate E. Burton. (2019). Radiotherapy[M]// Management of Adult Glioma in Nursing Practice.

[8] Antoine Aspeel, Damien Dasnoy, Raphael M. Jungers. (2019). Optimal Intermittent Measurements for Tumor Tracking in X-ray Guided Radiotherapy[C]// Image-Guided Procedures, Robotic Interventions, and Modeling.

[9] M. A. R. Loja, D. S. Craveiro, L. Vieira, et al. (2019). Radiotherapy-customized head immobilization masks: from modeling and analysis to 3D printing [J]. Nuclear Science and Techniques, 30(9).

[10] Ma Shumei, Liang Zhenzhen, Chen Qiao. (2019). The challenges of precision radiotherapy technology to the traditional theory of radiobiology [J]. Chinese Journal of Radiological Medicine and Protection, 39(8): 563-571.

[11] T Al-sudani, P Metcalfe, D Cutjar. (2019). Dose build up characteristics with eXaSkin bolus during 6MV radiotherapy: MOSkin dosimetry results [J]. Journal of Physics Conference Series, 1154(1): 012024.

[12] MA Guangdong and HONG Li. (2018). Review of Imaging Radiotherapy for Cervical Cancer [J]. China Medical Devices, 33(5): 117-129.

[13] Cao Fei, Zhao Yongliang, Li Ming, Liu Yu and Chu Kaiyue. (2019). Study on placement error and dosimetric difference of cervical cancer in supine and prone position [J]. Modern Oncology, 27(21): 3862-3865.

[14] Mason J H. (2018). Quantitative cone-beam computed tomography reconstruction for radiotherapy planning [M].

[15] Buckley J G, Wilkinson D, Malaroda A, et al. (2018). Investigation of the radiation dose from cone-beam CT for image-guided radiotherapy: A comparison of methodologies [J]. Journal of Applied Clinical Medical Physics, 19(1).

[16] Chen Caixia, Liu Libin and Jiang Jie. (2019). The influence of cone beam CT image guidance on radiotherapy positioning error after breast conserving surgery [J]. Medical Equipment, 32(19): 30.

[17] Yang H. J. (2019). Analysis of the accuracy of the positioning technique in radiotherapy of cervical cancer [J]. Cardiovascular Disease Electronic Journal of integrated traditional Chinese and Western Medicine, 7(16): 179-180.

[18] Xu Hong, Chen Liang, Zhang Jing, Lu Guohua. (2010). Review of the development of radiotherapy simulation [J]. China Foreign Medical Treatment, 28: 190-191.

[19] Leng Fu Lei. (2019). The comparative analysis of CBCT and EPID in radiotherapy of body tumor [J]. The Medical Journal of the Present Clinical, 32(02): 138. 
[20] Liu Bing, Zeng Zili, Wang Yongbing and Lin Feng. (2008). Study on the positioning error of cervical cancer in conventional radiotherapy by simulated positioning machine [J]. Medical Equipment, 22(1): 20-22.

[21] Han Yuan-yuan, Mao Kai, Dai Tian-tian, et al. (2019). Dosimetric comparison between volume-modulated arc therapy and intensity modulated radiation therapy for cervical carcinoma in prone position [J]. Journal of China-Japan Friendship Hospital, 33(5): 277-280.

[22] Xu Jian, Lin Hailong, Zou yunyun and Gong Wei. (2016). Analysis of the positioning errors of two kinds of fixators in intensity modulated radiotherapy for cervical cancer [J]. China Medical Device Information, 2016(12): 47-51.

[23] Zhang Shengfeng, Wang Xuetao, Chen yancan, Zhou Hanfen, Yang Geng, Zhao Shiwu, Zhu Yuanhu and Cai Chunya. (2015). Study on the accuracy of positioning technique in radiotherapy of cervical cancer [J]. Clinical Medicine \& Engineering, 22(3): 257-258.

[24] Li Ping, Chen minbin, Tang Min, Jin Jun, Zhou Lina, Zhang Yan, Yang agile, Zhu Shengchao, Lu Jingjing and Tianye. (2019). Analysis of setup error by cone beam computed tomography in intensity modulated radiotherapy for cervical cancer [J]. Chinese Clinical Oncology, 24(5): 440-444.

[25] Liu Yun, Yu Xian, Xiao He, Hu Nanand Wang Ge. (2016). Effect of body mass index on setup errors in cervical cancer treated with image-guided radiotherapy [J]. Journal of Third Military Medical University, 38(4).

[26] Liu Yi, Dai Xinguo and Wang lisen. (2019). The influence of image guided radiotherapy on the positioning error of cervical cancer patients [J]. Henan Medical Research, 28(14): 2538-2540.

[27] CAI Xuru, FANG Linming, JIN Cheng and CHEN Guofu. (2014). Study on effects of image guided techniques on setup error of cervical cancer in different body types [J]. China Modern Doctor, 52(33): 35-38.

[28] Saara Kares, Olga Veijalainen, Ivana Kholová. (2019). HIGH RISK HPV testing as the primary screening method in an organised regional screening programme for cervical cancer: The value of HPV 16 and HPV 18 genotyping? [J]. Apmis, 2019.

[29] Chen V E, Gillespie E F, Manger R P, et al. (2019). The impact of daily bladder filling on small bowel dose for intensity modulated radiation therapy for cervical cancer [J]. Medical Dosimetry, 44(2): 102-106.

[30] Wipperman J, Neil T, Williams T. (2018). Cervical Cancer: Evaluation and Management [J]. 97(7): 449-454.

[31] Bastien Rigaud, Antoine Simon, Maxime Gobeli. CBGGuided evolutive library for cervical adaptive IMRT [J]. Medical Physics, 2018, 45.

[32] Lu Ying and Song Jianbo. (2016). Bladder volume changes and set-up error during the course of intensity-modulated radiation therapy of cervical cancer [J]. Journal of Practical Medical Techniques, 23(9): 942-944.

[33] HE Ya, YANG Jin-ping, LIU Wei, et al. (2017). Investigation of fusion dose distribution for locally advanced cervical cancer under different bladder status with intensity-modulated radiotherapy combined with intracavitary brachytherapy [J]. Chinese Journal of Cancer Prevention \& Treatment, 2017.

[34] Caroline Lafond, Jairo Espinosa, Renaud de Crevoisier, et al. (2017). Quantification of dose uncertainties for the bladder in prostate cancer radiotherapy based on dominant eigenmodes [C]// 13th International Symposium on Medical Information Processing and Analysis.

[35] H. Zhang, S. Lu, J. Lang. (2019). Analysis of Local Control Rate and Toxicity of Radiotherapy Dose of Cervical Cancer [J]. Practical Journal of Clinical Medicine.

[36] Pobijakova M, Scepanovic D, Paluga M, et al. (2018). Dynamics of High Risk Clinical Target Volume reduction during Brachytherapy and impact on its coverage in patients with inoperable cervical cancer [J]. Neoplasma, 65(3): 425-430.

[37] Nesseler J P, Charra-Brunaud C, Salleron J, et al. (2017). Effect of bladder distension on doses to organs at risk in Pulsed-Dose-Rate 3D image-guided adaptive brachytherapy for locally advanced cervical cancer [J].

[38] Zhen X, Chen J, Zhong Z, et al. (2017). Deep convolutional neural network with transfer learning for rectum toxicity prediction in cervical cancer radiotherapy: a feasibility study [J]. Physics in Medicine \& Biology, 62(21): S168.

[39] Chakor Vora, Sudeep Gupta. (2019). Targeted therapy in cervical cancer [J]. ESMO Open, 2019, 3(Suppl 1).

[40] Delgado A B, Cohen D, Eng T, et al. (2016). SU-F-T-590: Modeling PTV Dose Fall-Off for Cervical Cancer SBRT Treatment Planning Using VMAT and Step-And-Shoot IMRT [J]. 2016, 43(6): 3599-3599.

[41] Sun R, Mazeron R, Chargari C, et al. (2016). CTV to PTV in cervical cancer: From static margins to adaptive radiotherapy [J]. Cancer/Radiothérapie, 20(6-7): 622-628. 